\title{
EDITORIAL
}

\section{Behavioural changes in temporal lobe epilepsy ${ }^{1}$}

\begin{abstract}
About 30 years ago pioneering studies, particularly by English investigators, began to alert clinicians to the existence of the behavioural changes of temporal lobe epilepsy. Yet despite this long history. disputes continue about the status of this syndrome. A major cause, although not the exclusive one, of these disagreements is that the phrase 'behavioural change in temporal lobe epilepsy' evokes differing associations. I shall present here briefly the view of one group and contrast these with some other conceptions.
\end{abstract}

According to this view a characteristic syndrome of behavioural change (Waxman \& Geschwind, 1975; Bear \& Fedio, 1977) occurs with higher frequency in temporal lobe epilepsy (and possibly in other cases with a spike focus in limbic structures) than in other neurological conditions. It is also more frequent in this condition than in other forms of epilepsy. The study of Bear \& Fedio (1977) brings evidence that it occurs in a very high percentage of temporal epileptics. It is commonly observed on first encounter and is therefore not primarily the result of drug therapy. It is also rare in other forms of epilepsy treated with the same drugs. Since it may precede the first recognized seizure and is rare in other neurological conditions, it is unlikely to be the result of stress of illness. Although some patients suffer from behavioural changes which occur during or after seizures, in most patients the characteristic syndrome is interictal, i.e. present constantly, without obvious relationship to individual seizures.

The syndrome includes the following features: increased concern with philosophical, moral or religious issues, often in striking contrast to the patient's educational background, an increased rate of religious conversions (or strongly justified, rather than casual, lack of religious feeling), hypergraphia (a tendency to highly detailed writing often of a religious or philosophical nature), hyposexuality (diminished sex drive sometimes associated with changes in sexual taste), and irritability of varying degree. Of particular interest was the finding in the Bear \& Fedio study of differences between those with left and right spike foci. The same personality picture can be seen in patients without temporal lobe foci but its high frequency in this type of epilepsy is remarkable. Furthermore, in an as yet unpublished study, Bear and his associates have found that these features were very much more frequent in temporal epileptics than in other cases of epilepsy.

Let me now underline certain features of this description. I have deliberately spoken of the behavioural or personality syndrome of temporal epilepsy, not of behaviour disorder. Many patients with the syndrome have neither been hospitalized nor in trouble with the police, and may make good adjustments, yet show the characteristic personality. Some patients with this constellation do, of course, have adjustment difficulties which bring them to medical attention. I have not been speaking of psychoses with temporal lobe foci. A small number of patients with this syndrome are psychotic, although even in these cases one usually observes the same fundamental personality pattern. Nor have I used the term schizophreniform. While some patients with idiopathic behaviour disturbances who would be labelled as schizoid or schizophrenic may possess some of the attributes of this syndrome, the majority of the patients with temporal epilepsy lack some of the cardinal features of schizophrenia, e.g. flatness of affect or blocking are unusual, even among those who are psychotic. Indeed, the use of terms borrowed from the standard psychiatric nomenclature may serve only to obscure the characteristic features of this condition.

Furthermore, I have not described what many physicians automatically assume is meant by behavioural change in epilepsy, i.e. the occurrence of periodic unmotivated aggression for which the patient is amnesic, and on the background of a normal personality; this is, in my experience, unusual. Most of these patients who act aggressively remember these episodes clearly and will justify their

1 Address for correspondence: Professor Norman Geschwind, Neurological Unit, Beth Israel Hospital, Boston, Mass., USA. 
behaviour. Furthermore, I have not found that prolonged fugue states are usually the result of temporal epilepsy (other causes, such as depression, being more common). Prolonged psychotic states with abrupt onset may occur in temporal epilepsy but these too are rare.

I have not argued that behaviour disorder of any type is more common in temporal epilepsy than in other epilepsies. In some American hospitals seizures following withdrawal (usually of alcohol) are by far the most common form of epilepsy. Since the patients involved are usually severe alcoholics they have, by the usual standards, a nearly $100 \%$ incidence of behaviour disorder, but their personality constellations are quite different from those in temporal epilepsy.

If this syndrome is so common why has it not been observed by others more frequently? It may be argued that personality change is common only in selected populations, e.g. patients in mental hospitals, or those referred to physicians with a special interest in the problem. The Bear \& Fedio study, however, was carried out on unselected patients being followed for epilepsy. I have repeatedly had the experience of demonstrating these personality features in patients whose records made no mention of personality change or indeed denied its presence. Most physicians do not enquire about a tendency to write poetry or keep a diary, or about religious conversions. Studies which do not look for this syndrome of course cannot find it. Thus, the study of Guerrant et al. (1962) claimed that 'behaviour disorder' was as common in hospital controls as in temporal lobe epileptics. They did not look for the syndrome described above. There were other methodological problems in this study. The frequency of functional behaviour disorder in the medical controls was $88 \%$, and $13 \%$ of these controls were diagnosed as psychotic by the psychiatrists. By contrast, on the basis of Minnesota Multiphasic Personality Inventory ratings, $23 \%$ of the psychomotor epileptics were rated as psychotic, but only $4 \%$ of the medical controls.

Unfortunately, when treatment of these personality changes is required, it is likely to be unsatisfactory. There has not been, to my knowledge, either a controlled study of medical treatment, nor are there pharmacologic studies of the type so often carried out on patients with manic-depressive illness or schizophrenia. No controlled study of surgery has been carried out. The impression gained from published studies is that temporal lobe surgery often reverses hyposexuality (which is sometimes replaced by dramatic hypersexuality) and often improves overall adjustment. On the other hand, the results in frankly psychotic patients are poor. Successful medical treatment of seizures often fails to alter the personality picture, which some believe often worsens as seizures diminish in number.

The unsatisfactory status of treatment for those who require it highlights the need for better understanding of pathogenesis. Disappointingly few animal experiments have been carried out. The clinical data, however, offer important clues. I have already noted the arguments against a major causative role of anticonvulsant drugs or psychological stress. The presence of damage (i.e. an ablative lesion) in the temporal lobe is probably not the cause, since anterior temporal lobe removal (which increases the size of the lesion) is often associated with improvement. Neither major nor minor seizures themselves appear to be the primary factor in producing the syndrome, since the intensity of personality change is poorly correlated with the frequency of fits.

The above list does not, however, exhaust all the possibilities. I have suggested that it is the presence of a spike focus which is the primary pathogenetic mechanism of the interictal change. A spike focus may produce few or many seizures, but it probably acts as an electrode discharging from time to time into limbic structures, thus altering their reactivity. Bear has used the evocative phrase 'the syndrome of sensory-limbic hyperconnection' to describe this mechanism. Thus, as Gastaut and Blumer have pointed out, bilateral temporal ablation leads to the Klüver-Bucy syndrome with its characteristic hypersexuality, while stimulation by a temporal focus leads to hyposexuality. This alteration in limbic reactivity to environmental stimuli could account for the characteristic deepening of the emotional life of these patients.

Recent findings in experiments on the phenomenon of kindling lend plausibility to this formulation. The electrical response in the amygdala increases as a series of intermittent stimuli (separated by intervals of 20 minutes or more) is applied. Eventually a major seizure may occur, which is followed by lowered reactivity of the focus to stimulation. Stevens \& Livermore (1978) have recently applied this experimental paradigm to the septal nuclei of monkeys. The kindled focus in this location does 
not lead to seizures but does produce marked behavioural change in the animals. Stevens (personal communication) has pointed out that everything which spikes is not epilepsy. It is possible that with increasing physiological knowledge we shall no longer speak of the personality syndrome of temporal lobe epilepsy, but rather of the syndrome of a limbic spike focus.

These animal findings point to new avenues of investigation. Kindling can be affected by many drugs not ordinarily considered to be epileptogenic or anticonvulsant, such as catecholamine and acetylcholine agonists and antagonists. Furthermore, drugs such as lidocaine, which generally act as anticonvulsants, may produce spikes in the amygdala. It is likely that we have been overlooking drugs which exert no anticonvulsant properties elsewhere, yet which act to suppress a limbic focus, or to prevent kindling.

Let me close by underlining the importance of this syndrome. We have no measures of its true overall frequency, nor how often the patients come first to psychiatric attention without any suggestion of the presence of epilepsy. It is, in any case, not rare. Increasing awareness of its existence and the use of more refined EEG and computerized radiological techniques may show it to be even more common than now appears to be the case. Even if it were relatively uncommon, it would be of major importance to the medical study of behaviour. If the pathogenesis suggested above is correct even in broad outline, then this syndrome will be unique in being the only cause of major behavioural change for which a reasonably detailed pathogenesis exists. Like Addison's disease, it would thus constitute a model disorder of basic physiological mechanisms. It could afford a means of better understanding and treatment of the neural disorders underlying other more frequent causes of behavioural change and gross maladjustment.

NORMAN GESCHWIND

\section{REFERENCES}

Bear, D. M. \& Fedio, P. (1977). Quantitative analysis of interictal behavior in temporal lobe epilepsy. Archives of Neurology 34, 454-467.

Guerrant, J., Anderson, W. W., Fischer, A., Weinstein, M. R., Jaros, R. M. \& Deskins, A. (1962). Personality in Epilepsy. Charles C. Thomas: Springfield, Ill.
Stevens, J. \& Livermore, A. (1978). Kindling of the mesolimbic dopamine system: animal model of psychosis. Neurology 28, 36-46.

Waxman, S. G. \& Geschwind, N. (1975). The interictal behavioral syndrome of temporal lobe epilepsy. Annals of General Psychiatry 32, 1580-1586. 